\title{
A HIGH REPETITION RATE VUV-SOFT X-RAY FEL CONCEPT*
}

\author{
J. Corlett" ${ }^{\#}$ J. Byrd, W. M. Fawley, M. Gullans, D. Li, S. M. Lidia, H. Padmore, G. Penn, \\ I. Pogorelov, J. Qiang, D. Robin, F. Sannibale, J. W. Staples, C. Steier, M. Venturini, S. Virostek \\ W. Wan, R. Wells, R. Wilcox, J. Wurtele, A. Zholents, LBNL, Berkeley, CA 94720, U.S.A.
}

\section{Abstract}

We report on design studies for a seeded FEL light source that is responsive to the scientific needs of the future. The FEL process increases radiation flux by several orders of magnitude above existing incoherent sources, and offers the additional enhancements attainable by optical manipulations of the electron beam: control of the temporal duration and bandwidth of the coherent output, reduced gain length in the FEL, utilization of harmonics to attain shorter wavelengths, and precise synchronization of the $\mathrm{x}$-ray pulse with seed laser systems. We describe an FEL facility concept based on a high repetition rate RF photocathode gun, that would allow simultaneous operation of multiple independent FEL's, each producing high average brightness, tunable over the VUV-soft $x$-ray range, and each with individual performance characteristics determined by the configuration of the FEL. SASE, enhanced-SASE (ESASE), seeded, harmonic generation, and other configurations making use of optical manipulations of the electron beam may be employed, providing a wide range of photon beam properties to meet varied user demands.

\section{SCIENTIFIC MOTIVATION}

Across the grand challenges in physics, chemistry, and materials science, an underlying theme is to understand, predict, and ultimately control the properties of matter. The LBNL program in R\&D toward advanced FELs addresses the needs for a next generation of x-ray radiation sources with new capabilities in photon pulse characteristics, which will allow study of phenomena inaccessible to either third-generation synchrotrons or other present-day sources.

The "emergent" properties of complex systems are of particular interest. Here, correlated interactions among charge carriers, and between charge carriers and constituent atoms, give rise to new properties and functionality with tremendous potential for practical applications. These same correlated interactions also challenge our understanding of complex systems in that they defy conventional paradigms. Direct quantitative measurements of the electronic and atomic structural dynamics on the ultrafast time scale of the underlying correlations will be indispensable in achieving new insight into the complex properties emerging from correlated phenomena in atoms, molecules, and solids. The fundamental time scales span:

- picoseconds, characteristic of conformational

\footnotetext{
* This work was supported by the Office of Science, U. S. Department of Energy, under Contract No.DE-AC02-05CH11231

\#jncorlett@lbl.gov
}

relaxations in molecular systems and electron-lattice energy transfer times in crystalline solids.

- 100 femtoseconds, characteristic of atomic vibrational periods in molecules and solids.

- $\sim 10$ femtoseconds, characteristic of electron-electron scattering times in solids.

- 100 attoseconds, characteristic of electron-electron correlations and valence electron motion.

The needs to directly probe electronic structure and dynamics demand a focus on the VUV and soft $\mathrm{x}$-ray regions, and the creation of experimental facilities that complement those being constructed with hard x-ray capabilities.

\section{OVERVIEW OF FACILITY CONCEPT}

High repetition rate and high average photon flux are essential to many experimental techniques. To meet these needs, we envision a facility comprised of a high bunch repetition rate $(\sim \mathrm{MHz})$, low-emittance and low energy spread RF photocathode electron gun, and a low-energy $(\sim 2 \mathrm{GeV})$ superconducting linac, feeding an array of approximately ten FELs through an elaborate beam switchyard. Each FEL operates independently at a repetition rate of $\sim 100 \mathrm{kHz}$. Photon energies would span approximately $10 \mathrm{eV}$ to $1 \mathrm{keV}$, with the possibility to reach higher photon energy at the expense of reduced photon flux. A variety of seeded and SASE FELs provide output radiation with a peak power from a few hundred megawatts to a few gigawatts. The temporal coherence available in the seeded FEL allows close to transformlimited x-ray pulses, resulting in a narrow bandwidth signal that may be utilized in experiments without a monochromator. Techniques have also been developed to use produce $\mathrm{x}$-ray pulses of a few hundred attosecond duration.

Figure 1 shows a schematic of a multi-user FEL facility concept. The major components are: (1) a low-emittance, low energy spread RF photocathode electron gun operating in CW mode at an RF frequency of $\sim 100 \mathrm{MHz}$, providing electron bunches at up to $\mathrm{MHz}$ repetition rate, (2) hardware for manipulating the electron-beam emittance in preparation for the FEL process, (3) a CW superconducting RF linac, (4) a beam switching system (5) multiple independent FELs and beamlines, and (6) lasers and optical systems for the photocathode gun, FEL seeding, pump-probe experiments, and timing and synchronization. A low-energy linac is used to minimize costs. The electron beam is dumped at the end of each FEL as we do not currently believe the added cost and complexity of electron beam recirculation and energy recovery is worthwhile for a machine of modest electron beam power. 
(5) Array of configurable FELs

Independent control of wavelength, pulse duration, polarization

Configured with an optical manipulation technique; seeded, attosecond, ESASE

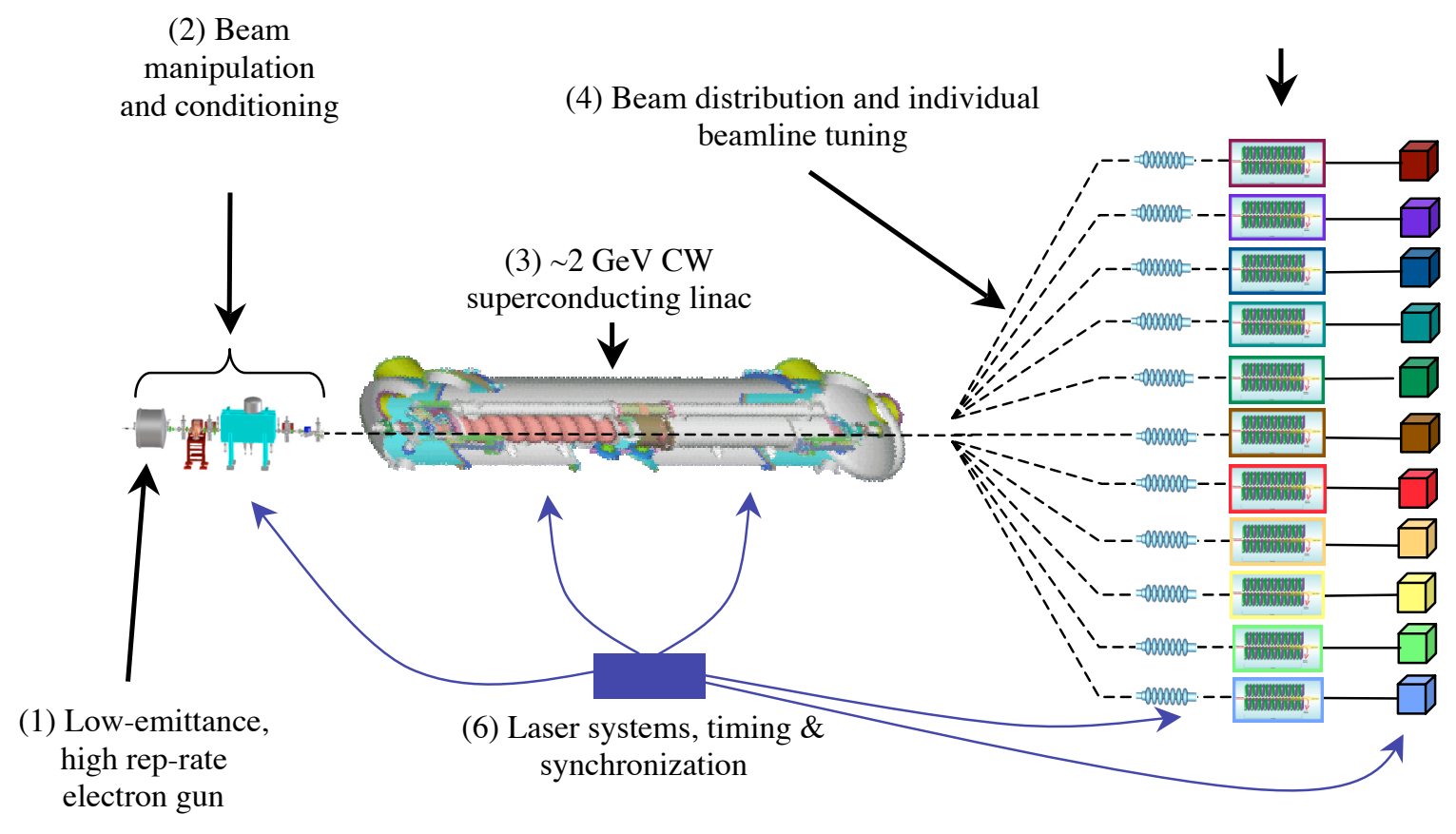

Figure 1: Schematic layout of a high repetition-rate, VUV - soft x-ray, seeded FEL facility

\section{CURRENT R\&D ACTIVITIES}

Our strategy is to address fundamental challenges, which are the cost-drivers and performance limitations of FEL facilities, and to develop critical technologies to enable full use of future facilities. R\&D activities are aimed at investigating accelerator physics and technologies in key areas:

- Theoretical study, modeling, and experimental development of low emittance, high quantum efficiency cathodes.

- Design studies of RF accelerating structures for application in photocathode guns producing high repetition-rate (of order $\mathrm{MHz}$ ), low emittance beams.

- Design studies of electron beam delivery systems, including emittance manipulations, high-resolution modeling of 6-D phase space, and low-emittance beam transport.

- Design studies of optical manipulations of electron beams for seeded and SASE FELs, providing short $\mathrm{x}$ ray pulses of variable duration, synchronous with the seed and pump laser sources, and also long transformlimited pulses with a narrow bandwidth. Design studies of means for production of attosecond x-ray pulses at various wavelengths.

- Development of ultra-stable optical distribution systems based on optical fiber, and using $\mathrm{CW}$ optical signal transmission, for precise timing and synchronization of accelerator and laser systems.

\section{Photocathode design}

The aim of this program is to improve the beam quality through development of a cathode that produces a beam with very low thermal emittance. We also aim to increase the quantum efficiency up to the point at which conventional laser technology can be used to produced tailored electron pulses at $\mathrm{MHz}$ repetition rate. As a first step, we have investigated metallic photocathodes, and determined that photo-current at the very low photon energies typically used is dominated by surface states. This directly has led to a prediction of the minimum transverse momentum, and to a direction for producing lower emittance though use of other crystalline surfaces. These studies will be extended to metallic systems in which the surface electric field is manipulated using plasmonic interactions, and to semiconductor systems. The latter have the advantage that some degree of thermalization can take place, resulting in colder emission. This work is based on understanding the near Fermi surface electronic structure, through use of very low energy photoelectron spectroscopy, and through electronic structure modeling. In addition, we have studied the impact on beam phase space of the stochastic Coulomb scattering of electrons following emission from the cathode surface [1]. 


\section{$R F$ gun design}

We are developing a design for a high-brightness, highrepetition rate electron gun that uses a normal conducting RF structure in the VHF range, between 50 to $100 \mathrm{MHz}$. The gun cavity has a strongly re-entrant geometry, with a coaxial center conductor at the end of which the cathode is mounted. The lower frequency results in a larger cavity compared to the more common designs operating at $\sim 1$ to $\sim 3 \mathrm{GHz}$. A significant benefit of using a larger cavity is a dramatic reduction of the power density on the cavity walls, which allows CW operation of the gun, and thus a bunch repetition rate of up to the RF frequency (dependent on photocathode-laser time structure). The photocathode can be embedded in a magnetic field to provide correlations useful for emittance exchange. Beam dynamics simulations indicate that normalized emittances smaller than $1 \mathrm{~mm}$-mrad are possible with gap voltage of $750 \mathrm{kV}$, with fields up to $20 \mathrm{MV} / \mathrm{m}$ at the photocathode, for 1 nanocoulomb charge per bunch after acceleration and emittance compensation. More details of the cavity design may be found in [2].

\section{Electron beam delivery systems}

Production of electron beams for x-ray FELs is a difficult and elaborate process consisting of the electron generation, acceleration, compression, and transport. A significant understanding of the underlying physics such as space charge effects, wake-fields, and coherent synchrotron radiation (CSR) has been gained over the past decade. Radical improvements in the electron beam quality may be needed to be able to build a cost effective VUV-soft $x$-ray FEL facility. We are developing understanding of the beam phase space evolution, and means to control and manipulate emittances, using both theoretical approaches and high-resolution numerical modeling. We have developed a parallel code suite, IMPACT, for advanced supercomputer modeling of high intensity, high brightness beams in rf linacs and photoinjectors [3]. An example is provided by a 100 million macroparticle simulation of the microbunching instability, that cannot be sensibly performed on today's single-processor computers. Multiprocessor simulations using IMPACT show the sensitivity to macroparticle number of the evolution of the microbunching instability. We are augmenting our present particle loading approach with "quiet-start" techniques, and in parallel we are also developing a direct Vlasov-Maxwell solution [4].

Following the accelerator, electron beams will be switched into each FEL in the array, in a time-sliced manner dependent on user needs. Techniques for switching the electron beam between FEL's are being studied, using pulsed ferrite magnets in a linear array, selectively switching the beam into the FEL beamlines.

\section{FEL design}

Our goal is to develop design concepts for flexible photon beam performance, based on a number of FEL configurations, fed by a low-energy electron accelerator that would provide experimentalists with a variety of options. FEL output capabilities under study include high flux time-domain pulses of femtosecond to hundredattosecond duration for pump-probe experimentation, and close to transform limited pulses of hundreds of femtosecond duration providing high energy-resolution with meV bandwidth. SASE, seeded, regenerative amplifier, and oscillator techniques are being simulated and developed, using GINGER and GENESIS simulation codes. Start-to end modeling of the electron beam is critical in determining realistic FEL performance capabilities, and these studies are performed in conjunction with development of beam delivery systems.

\section{Timing and synchronization systems}

To produce an ultra-stable timing and synchronization system with jitter reduced to the few femtosecond level, we have developed a laser-based scheme with optical signals distributed over a stabilized optical fiber [5]. Transmitting precise frequency and timing signals to allow synchronization of lasers and accelerator systems, over distances of hundreds of meters, is accomplished by measuring the phase delay in an optical fiber and actively compensating for differences with a piezoelectric modulator. At present, a $4 \mathrm{~km}$ fiber link has been stabilized to the femtosecond level. $2 \mathrm{~km}$ of fiber in this link passes under several roads and through several buildings at LBNL, demonstrating that the fiber stabilization system is robust under real-world conditions. Techniques for synchronization of laser systems using CW optical signals, propagated over stabilized optical fiber links, are described in [6].

\section{REFERENCES}

[1] J. Qiang, et al, "Numerical study of Coulomb Scattering Effects on Electron Beam from a Nanotip" TUPMN116, this conference.

[2] J. W. Staples, et al, "Design of a VHF-band RF Photoinjector with Megahertz Beam Repetition Rate", THPMN117, this conference.

[3] J. Qiang, I. V. Pogorelov, R. Ryne, "Parallel Beam Dynamics Simulation Tools for Future Light Source Linac Modeling", THPAS007, this conference.

[4] I. V. Pogorelov, et al, "Simulation of the Microbunching Instability in Beam Delivery Systems for Free Electron Lasers", TUPMN114, this conference.

[5] R. B. Wilcox, J. W. Staples, and J. M. Byrd, "Demonstration of femtosecond phase stabilization in $2 \mathrm{~km}$ optical fiber", MOPAS028, this conference.

[6] R. B. Wilcox and J. W. Staples, "Systems design concepts for optical synchronization in accelerators", FROAC05, this conference. 